\title{
Time-dependent response of intact intervertebral disc - In Vitro and In-Silico study on the effect of loading mode and rate
}

\author{
Mohammad Nikkhoo ${ }^{a, b^{*}}$, Ya-Wen Kuo ${ }^{b}$, Yu-Chun Hsu ${ }^{b}$, Kinda Khalaf ${ }^{c}$, Mohammad \\ Haghpanahi ${ }^{\mathrm{d}}$, Mohamad Parnianpour ${ }^{\mathrm{e}}$ and Jaw-Lin Wang ${ }^{\mathrm{b}}$
}

${ }^{a}$ Department of Biomedical Engineering, Science and Research Branch, Islamic Azad University, Tehran, Iran
${ }^{b}$ Institute of Biomedical Engineering, College of Medicine and Engineering, National Taiwan University, Taipei, Taiwan
${ }^{c}$ Department of Biomedical Engineering, Khalifa University of Science, Technology and Research, Abu Dhabi, UAE
${ }^{d}$ School of Mechanical Engineering, Iran University of Science and Technology, Tehran, Iran
${ }^{e}$ Department of Industrial \& Manufacturing, University of Wisconsin, Milwaukee, USA

ARTICLE INFO ABSTRACT

Article history:

Received March 6, 2014

Accepted 28 November 2014

Available online

28 November 2014

Keywords:

Porcine intervertebral disc

Time-dependent response

Poroelastic FE model

Creep

Cyclic loading

Response surface methodology

\begin{abstract}
The investigation of dynamic response of intervertebral disc is beneficial for the development of new synthetic and engineered tissues for treating diseased or injured disc. There are limited experimental studies on comparing the effect of loading mode and rate on global response of intervertebral disc. In this study, in-vitro experiments were performed using a total of 24 porcine motion segments. The harvested specimens were assigned to prolong and 2 different cyclic loadings. Both disc deformations and water contents were measured to investigate how the mode and rate of loading affect the response of intervertebral disc. In parallel, a backward FE poroelastic model combined with in-vitro experiments were used to find the material properties of intervertebral discs. The experimental result showed that the final disc height loss under creep loading was significantly greater than cyclic groups. Increasing the frequency of cyclic loading decreased the disc height loss. The water content decreased significantly in cyclic loading from those in prolong loading. The backward FE models showed that, the elastic modulus of anulus fibrosus and nucleus pulposus were $2.43( \pm 0.48) \mathrm{MPa}$ and $1.46( \pm 0.29)$ $\mathrm{MPa}$, respectively. The hydraulic permeability was $2.08( \pm 0.42) \times 10^{-16} \mathrm{~m}^{4} / \mathrm{Ns}$, and the Poisson's ratio was $0.21( \pm 0.03)$. In conclusion, this study investigated how the loading mode and rate affect porcine intervertebral disc deformation. It is found that dynamic stiffness is greater at higher frequencies which resulted from interactions between the solid phase and fluid flow within the disc.
\end{abstract}

\section{Introduction}

The intervertebral disc plays the essential biomechanical roles of supporting load, absorbing shock and providing spinal stability. It is composed of a nucleus pulposus bounded by anulus fibrosus and endplates. Regarding to the nature of intervertebral disc, its mechanical properties varies under

* Corresponding author. Tel.: +98-21-44474321

E-mail addresses: $\mathrm{m}$ nikkhoo@hotmail.com (M. Nikkhoo) 
prolong and repetitive loadings. During the repetitive loading, the fluid of disc can be squeezed out and in resting duration, the fluid content of disc can be restored, but may not be able to heal the disc in short time. The inequality of loading or rest may be the reason that both reversible and irreversible changes of disc mechanical properties following cyclic loading (Wang et al., 2008). Moreover, the time-dependent response of disc depends on the degree of disc integrity and hydration.

The investigation of dynamic response can be beneficial for the development of new synthetic and engineered tissues for treating diseased or injured intervertebral disc (Kuo \& Wang, 2010). The knowledge of time-dependent response of intervertebral disc can be valuable to enhance understanding of changes in material properties during disc hydration, degeneration severity and therapeutic interferences. Understandings of the motion segment behaviors under prolong and cyclic loading conditions are a priority for defining safe loading regimens in design of instrumentations and implants.

The intervertebral disc displays poroelastic characteristics. Many experimental observations recommended that it is more realistic to consider intervertebral disc as a multiphase material. Many physical models were developed to simulate this phenomenon. The biphasic theory developed by Mow et al. (Mow et al., 1980) considered the soft tissues as a mixture of solid phase and fluid. Simon et al. (1985) modeled the intervertebral disc using poroelastic theory. Later, different poroelastic finite element models were developed for distinct structural regions (Argoubi \& Shirazi-Adl, 1996; Williams et al., 2007; Schmidt et al., 2010).

There are few experimental studies on comparing the effect of loading mode and rate on global response of intervertebral disc. Furthermore, there is lack of knowledge about the definition of poroelastic material properties of the anulus fibrosus and nucleus pulpous in both prolong and cyclic loading. Hence, the objective of this study was first to study the time-dependent response of intervertebral disc and second, to extract relative poroelastic material properties. In-vitro and in-silico studies on porcine disc model were performed to investigate how the mode and rate of loading affect the response of intervertebral disc.

\section{Materials and methods}

\subsection{In-vitro Experimental Test}

A total of 24 porcine thoracic motion segments were dissected from 6-month-old juvenile pigs immediately after death. Each specimen was carefully cleaned of the soft tissues and posterior elements and facet joints were carefully dissected. The upper half of cranial vertebra and lower-half of caudal vertebra were also removed. The 2 cutting surfaces were parallel aligned to warrant pure axial compressive deformation. Specimens were wrapped in phosphate buffer saline (PBS) soaked gauze, sealed in plastic bag, and stored at -20 degree Celsius in a refrigerator until experiment. The disc did not receive any forms of injury.

The harvested specimens were assigned to one of the three groups and underwent one of loading protocols (Fig. 1) using a mechanical testing apparatus (ElectroForce ${ }^{\circledR}$ 3510, Bose Corporation, $\mathrm{MN})$. The average radius, height and cross-sectional area were measured which were $13.28( \pm 0.32)$, $5.0( \pm 0.29) \mathrm{mm}$ and $512.3( \pm 51.16) \mathrm{mm}^{2}$, respectively. The radius of the disc was simplified to be the geometric average of the long and short axes of the porcine disc. Specimens were mounted in center of a home-made chamber filled with PBS solution to mimic the fluid inflow and outflow during loading and unloading (Fig. 2). A Teflon plate was attached to the actuator of the material testing machine to transfer the applied loading to the specimen. After preloading, each specimen was subjected to its assigned main loading protocol, and displacement versus time was recorded. 


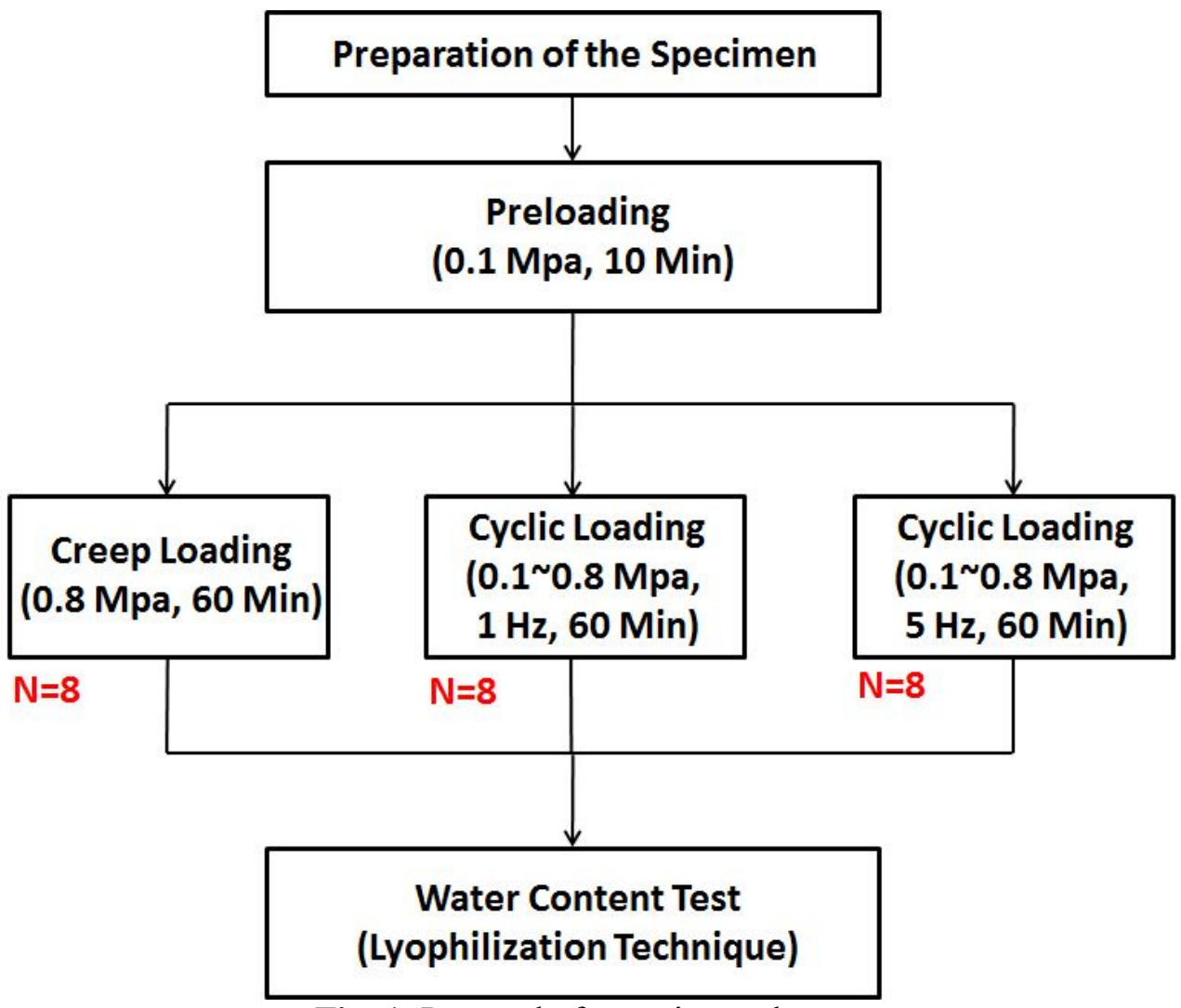

Fig. 1. Protocol of experimental tests

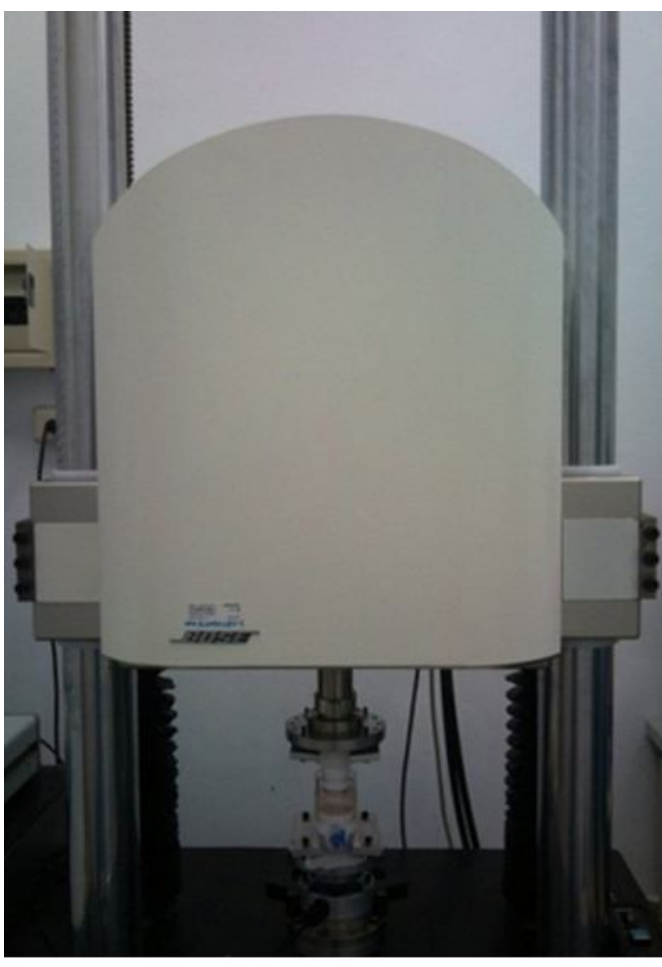

(A)

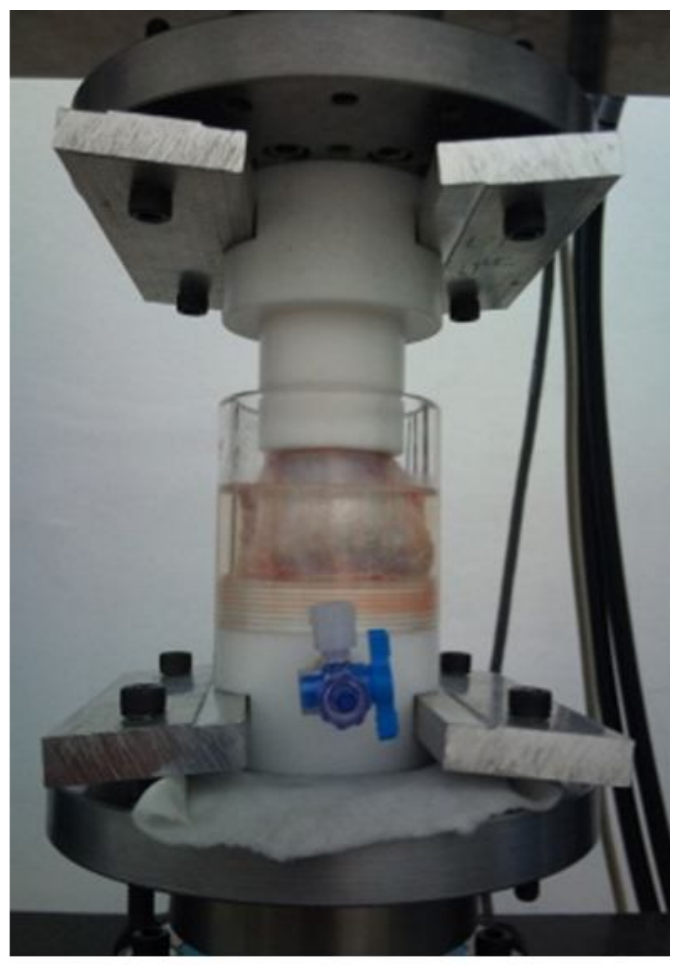

(B)

Fig. 2. (A) The mechanical testing apparatus and (B) home-made chamber to mimic the fluid inflow and outflow during loading and unloading 
Immediately after mechanical test, each specimen was cut from the midline. To measure the water content of intervertebral disc in different regions, six specimens were cut from nucleus pulposus ( 2 specimens), inner anulus fibrosus ( 2 specimens), and outer anulus fibrosus ( 2 specimens). The specimens were weighted and then dried using lyophilization technique. The dried specimens were weighted again to evaluate the water content.

\subsection{Backward Poroelastic FE Modeling}

The structures of intervertebral disc can be replicated as an elastic medium containing interconnected fluid-saturated pores using poroelastic continuum mechanics (Simon et al., 1985). The principal equations for a porous media are based on the equilibrium of the solid and fluid phase (Biot, 1941). The momentum balance of the solid phase is as follows (Kojic et al., 1998),

$$
(1-n) \nabla^{T} \sigma_{s}+(1-n) \rho_{s} \mathbf{b}+\mathbf{k}^{-1} n \mathbf{q}-(1-n) \rho_{s} \ddot{\mathbf{u}}=0
$$

where $n$ is porosity, $\sigma_{\mathbf{s}}$ is stress in the solid phase, $\rho_{s}$ is density of the solid, $\mathbf{b}$ is body force per unit mass, $\mathbf{k}$ is hydraulic permeability matrix, $\mathbf{q}$ is the relative fluid velocity $\left(\mathbf{q}=\mathrm{n}\left(\mathbf{v}_{f}-\dot{\mathbf{u}}\right)\right)$, and $\ddot{\mathbf{u}}$ is acceleration of the solid phase. The equivalent of momentum of the fluid phase, which is also recognized as the generalized Darcy's law (Kojic et al., 1998), is as follows:

$$
-\mathrm{n} \nabla p+n \rho_{f} \mathbf{b}-\mathbf{k}^{-1} n \mathbf{q}-n \rho_{f} \dot{\mathbf{v}}_{f}=0
$$

where $p$ is pore fluid pressure, $\rho_{f}$ is fluid density, $\mathbf{v}_{f}$ is fluid velocity, and $\dot{\mathbf{v}}_{f}$ is fluid acceleration. The solid matrix deforms and the fluid flows within the solid matrix. The fluid flow from higher pressure to lower pressure alters the stress and strain field of the matrix. The friction between solid and fluid makes the behaviour of the material to be rate-dependent.

Based on saturated porous media theory, a general disc poroelastic FE model of intervertebral disc was developed using ABAQUS v6.9 (SIMULIA, Providence, RI, USA). This model was previously validated (Nikkhoo et al., 2013a) by in-vitro human disc experiments for both creep (Heuer et al., 2007) and cyclic loading ( $\mathrm{Li}, 1994)$. With the aim of using the model for investigation of the porcine tests, the geometry and initial conditions of water content were modified for porcine disc. For this purpose, the radii, disc heights and porosities of 24 simulated discs were chosen from measurements in the individual tested discs. 8-node axisymmetric elements with quadratic interpolation of displacement field and linear interpolation of pore pressure were used. The numbers of nodes and elements were 19916 and 6517, respectively. Nonlinear large deformations were used for calculation (Nikkhoo et al., 2011; Nikkhoo et al., 2013c).

The inferior surface of the lower vertebra was fixed. The axial displacement of the superior surface of upper vertebra was assumed to be consistent. The fluid was free to flow and no element was allowed to slide along the interface of different materials. The swelling phenomenon was mimicked by imposing a fixed boundary pore pressure (BPP) (Galbusera et al., 2011) on the external surfaces of intervertebral disc. The value of the BPP was chosen $0.3 \mathrm{MPa}$ as it yielded the best agreement for the reduction of both disc height loss and pore pressure in validation procedure in our previous study (Nikkhoo et al., 2013a).

A linear ramp from 0 to $0.1 \mathrm{MPa}$ over 60 seconds followed by a 10 -min unconfined loading was applied on the superior surface of upper vertebra as preloading. Furthermore, each disc simulation was subjected to its assigned main loading protocol (Fig. 1). 
A validated backward FE algorithm and quadratic response surface (QRS) regression (Nikkhoo et al., 2013c) were used to calculate the optimal sets of mechanical properties for intact intervertebral disc during different types of loading (i.e. prolong loading and cyclic loading with two different frequency). For this purpose, three main material properties, elastic modulus $(E)$, hydraulic permeability $(k)$, and Poisson's ratio $(v)$ were chosen as the independent variables of the intervertebral disc FE model. To simplify the optimization procedure, the ratio of the elastic modulus for the anulus fibrosus and nucleus pulposus was assumed to be 1.67 and the ratio of hydraulic permeability were assumed to be 1 during analysis (Argoubi \& Shirazi-Adl, 1996; Schmidt et al., 2010).

A full factorial design of experiment (DOE) for the FE simulation was conducted using MATLAB (Mathworks, Inc., Natick, MA, USA). Three factors, i.e., $E, k$ and $v$, and 3 levels in each factor resulted in 27 input combinations sets. Based on our previous studies (Nikkhoo et al., 2013c;b), the initial ranges of independent parameters were set (Table 1). Twenty seven simulations were performed for each specimen. QRS model was constructed and optimized to find the best set independent variables. The results were considered to have sufficient accuracy when the normalized percentage of the root mean square error was less than $7.5 \%$.

Table 1. Three levels of independent variables which were chosen as initial values for backward FE model

\begin{tabular}{llll}
\hline Independent Variable & Low Level & Medium Level & High Level \\
\hline Elastic Modulus $(\mathrm{AF}) *(\mathrm{MPa})$ & 1.5 & 2.25 & 3 \\
Hydraulic Permeability $\left(\mathrm{m}^{4} / \mathrm{Ns}\right)$ & $1 \times 10^{-16}$ & $1.75 \times 10^{-16}$ & $2.5 \times 10^{-16}$ \\
Poisson's Ratio & 0.1 & 0.2 & 0.3 \\
\hline $\begin{array}{l}\text { *Elastic modulus of anulus fibrosus was selected as independent variable and the elastic modulus of nucleus pulposus was calculated based on the } \\
\text { proposed constant ratio (EAf }\end{array}$
\end{tabular}

\subsection{Statistical Analysis}

One-way ANOVA (SPSS, SPSS Inc., Chicago, IL, USA) was used to test the effect of loading type on disc height loss and fluid loss during the prolong and cyclic loading regimes. As well, the effect of loading rate on dynamic response of IVD was evaluated with the paired $t$ test (SPSS, SPSS Inc., Chicago, IL, USA).

\section{Results and discussion}

This study presented in-vitro and in-silico studies on porcine disc model to investigate how the mode and rate of loading affect the response of intervertebral disc. The result of in-vitro experiment showed that the final disc height loss under creep loading was significantly greater than cyclic groups (Fig. 3). Increasing the frequency of cyclic loading decreased the disc height loss (Fig. 4a). Dynamic stiffness of disc was $2.35( \pm 0.27)$ for $1 \mathrm{~Hz}$ group which significantly increased to $3.03( \pm 0.3) \mathrm{MN} / \mathrm{m}$ for $5 \mathrm{~Hz}$ group (Fig. 4b). The water content decreased significantly in cyclic loading from those in prolonged loading as shown in Fig. 5.

The individual disc deformations during different types of loading conditions were well fitted by FE model predictions. The average normalized percentages of normalized root mean square error were $4.87( \pm 0.96) \%, 5.37( \pm 1.21) \%$ and $6.02( \pm 1.33) \%$ for three loading conditions. The backward FE models showed that, the elastic modulus of anulus fibrosus and nucleus pulposus were 2.43 $( \pm 0.48) \mathrm{MPa}$ and $1.46( \pm 0.29) \mathrm{MPa}$, respectively. The hydraulic permeability was $2.08( \pm 0.42) \times 10^{-}$ ${ }^{16} \mathrm{~m}^{4} / \mathrm{Ns}$, and the Poisson's ratio was $0.21( \pm 0.03)$. 


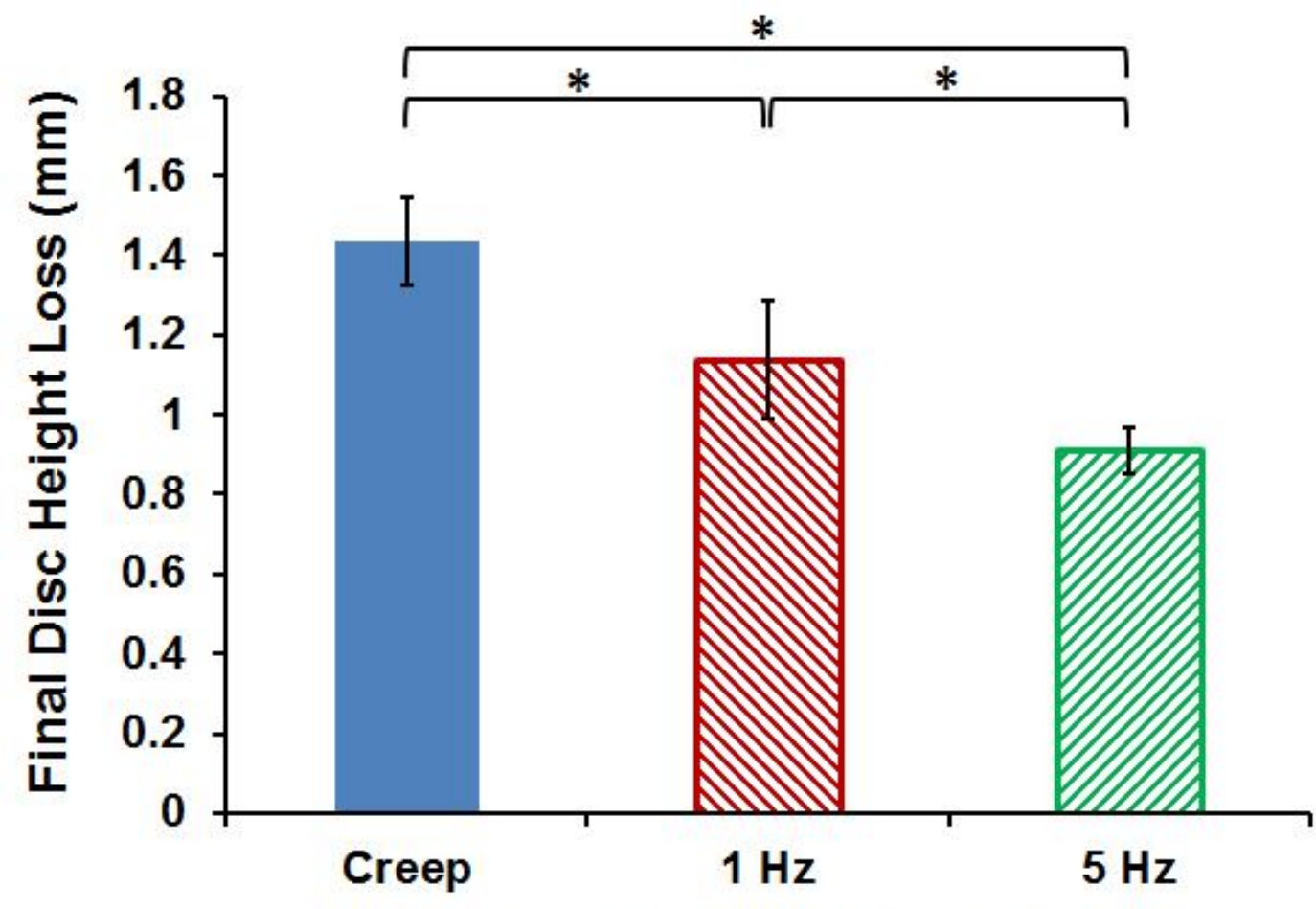

Different Loading Conditions

Fig. 3. Effect of loading mode on disc height loss

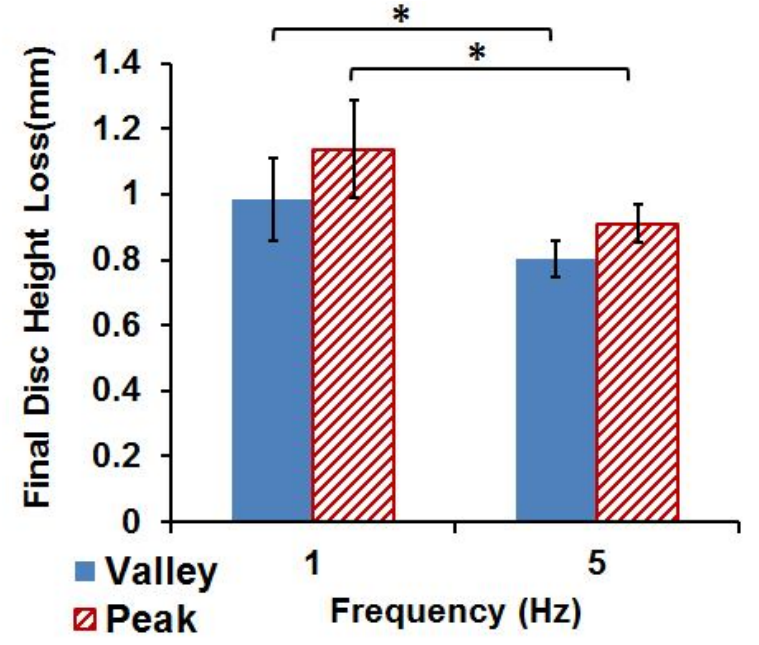

(A)

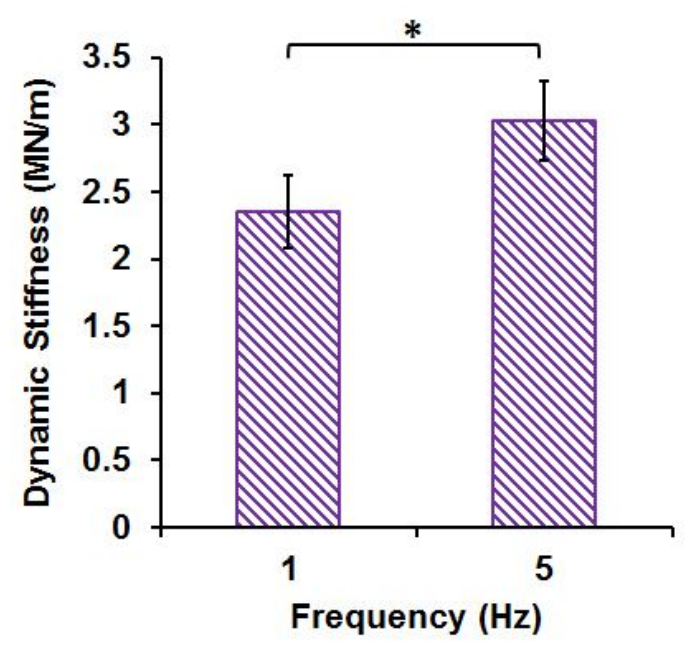

(B)

Fig. 4. Comparison of rate-dependent dynamic response of intervertebral disc

Some limitations of current study should be considered. Healthy porcine discs were used instead of healthy human discs. Accessing a large number of healthy human disc specimens is so difficult and the available cadaveric human disc specimens are usually degenerated. Porcine disc is comparable to the human one in different aspects of histology, geometry, and the mechanical properties (McLain et al., 2002; Beckstein et al., 2008). For simplification of the FE analysis, the posterior bony parts, the facet joints, and the ligaments were neglected. This can be approximately justified due to the negligible effects of the posterior elements under pure axial compression loadings. Although the 
elastic modulus and hydraulic permeability of human anulus fibrosus is direction-dependent, isotropic values were chosen for poroelastic model. This assumption was considered to make the optimization procedure feasible. Further complex studies could be performed to determine combined anisotropic parameter effects.

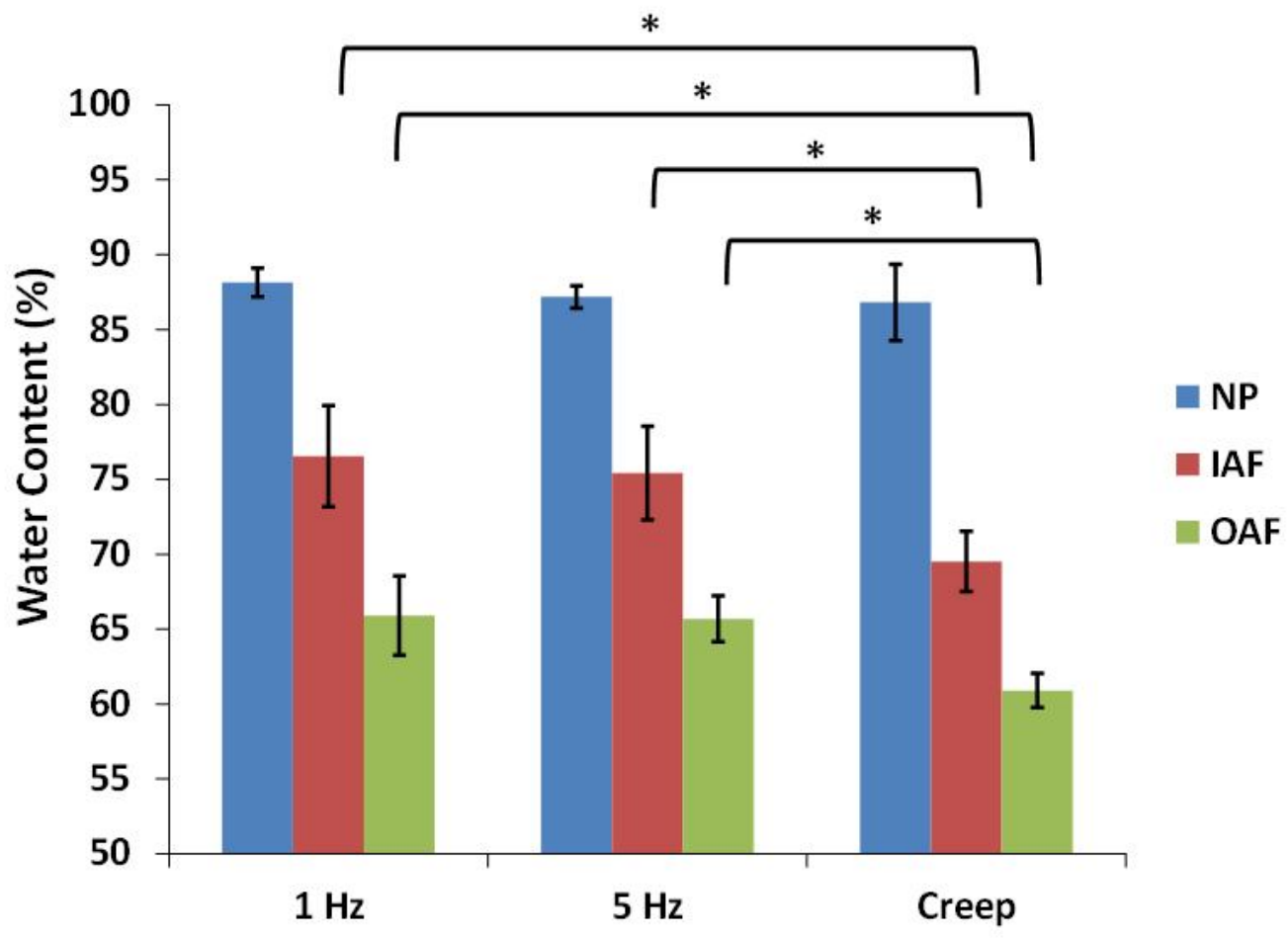

Fig. 5. Effect of loading mode and rate on disc fluid loss

\section{Conclusion}

This study investigated how the loading mode and rate affect porcine intervertebral disc deformation. We found that dynamic stiffness is greater at higher frequencies which resulted from interactions between the solid phase and fluid flow within the disc. This study also combined FE simulations, in-vitro experiments, and QRS regression to provide a suitable set of material properties for poroelastic FE modeling of intervertebral disc. The material properties, loading and boundary conditions are the critical issues in finite element modelling of the soft tissues, which can provide detailed findings in spine researches.

\section{Acknowledgement}

This study was funded by National Science Council, Taiwan (NSC 101-2628-B-002-039-MY3, 101-2221-E-002-060-MY3, 99-2628-B-002-039-MY3).

\section{References}

Argoubi, M., \& Shirazi-Adl, A. (1996). Poroelastic creep response analysis of a lumbar motion segment in compression. Journal of biomechanics, 29(10), 1331-1339.

Beckstein, J. C., Sen, S., Schaer, T. P., Vresilovic, E. J., \& Elliott, D. M. (2008). Comparison of animal discs used in disc research to human lumbar disc: axial compression mechanics and glycosaminoglycan content. Spine, 33(6), E166-E173. 
Biot, M. A. (1941). General theory of three-dimensional consolidation. Journal of applied physics, 12(2), 155-164.

Galbusera, F., Schmidt, H., Noailly, J., Malandrino, A., Lacroix, D., Wilke, H. J., \& Shirazi-Adl, A. (2011). Comparison of four methods to simulate swelling in poroelastic finite element models of intervertebral discs. Journal of the mechanical behavior of biomedical materials, 4(7), 1234-1241.

Heuer, F., Schmitt, H., Schmidt, H., Claes, L., \& Wilke, H. J. (2007). Creep associated changes in intervertebral disc bulging obtained with a laser scanning device. Clinical Biomechanics, 22(7), 737-744.

Kojic, M., Filipovic, N., Vulovic, S., \& Mijailovic, S. (1998). A finite element solution procedure for porous medium with fluid flow and electromechanical coupling. Communications in numerical methods in engineering, 14(4), 381-392.

Kuo, Y. W., \& Wang, J. L. (2010). Rheology of intervertebral disc: an ex vivo study on the effect of loading history, loading magnitude, fatigue loading, and disc degeneration. Spine, 35(16), E743E752.

Li, S. (1994). Response of human intervertebral discs to prolonged axial loading and low-frequency vibration. University of Illinois at Chicago

McLain, R. F., Yerby, S. A., \& Moseley, T. A. (2002). Comparative morphometry of L4 vertebrae: comparison of large animal models for the human lumbar spine. Spine, 27(8), E200-E206.

Mow, V. C., Kuei, S. C., Lai, W. M., \& Armstrong, C. G. (1980). Biphasic creep and stress relaxation of articular cartilage in compression: theory and experiments. Journal of biomechanical engineering, 102(1), 73-84.

Nikkhoo, M., Haghpanahi, M., Wang, J.L., and Parnianpour, M. (2011). Axisymmetric Poroelastic FE Modeling of Intervertebral Disc for Investigation of Lumbar Spine Biomechanics. Iranian Journal of Biomedical Engineering 5, 21-32.

Nikkhoo, M., Haghpanahi, M., Parnianpour, M., \& Wang, J. L. (2013a). Dynamic responses of intervertebral disc during static creep and dynamic cyclic loading: a parametric poroelastic finite element analysis. Biomedical Engineering: Applications, Basis and Communications, 25(01).

Nikkhoo, M., Hsu, Y. C., Haghpanahi, M., Parnianpour, M., \& Wang, J. L. (2013b). Material Property Identification of Artificial Degenerated Intervertebral Disc Models-Comparison of Inverse Poroelastic Finite Element Analysis with Biphasic Closed Form Solution. Journal of Mechanics, 29(04), 589-597.

Nikkhoo, M., Hsu, Y. C., Haghpanahi, M., Parnianpour, M., \& Wang, J. L. (2013c). A meta-model analysis of a finite element simulation for defining poroelastic properties of intervertebral discs. Proceedings of the Institution of Mechanical Engineers, Part H: Journal of Engineering in Medicine, 0954411913480668.

Schmidt, H., Shirazi-Adl, A., Galbusera, F., \& Wilke, H. J. (2010). Response analysis of the lumbar spine during regular daily activities - a finite element analysis. Journal of biomechanics, 43(10), $1849-1856$

Simon, B. R., Wu, J. S. S., Carlton, M. W., Kazarian, L. E., France, E. P., Evans, J. H., \& Zienkiewicz, O. C. (1985). 1985 Volvo Award in Biomechanics: Poroelastic Dynamic Structural Models of Rhesus Spinal Motion Segments. Spine, 10(6), 494-507.

Wang, J. L., Wu, T. K., Lin, T. C., Cheng, C. H., \& Huang, S. C. (2008). Rest cannot always recover the dynamic properties of fatigue-loaded intervertebral disc. Spine, 33(17), 1863-1869.

Williams, J. R., Natarajan, R. N., \& Andersson, G. B. (2007). Inclusion of regional poroelastic material properties better predicts biomechanical behavior of lumbar discs subjected to dynamic loading. Journal of biomechanics, 40(9), 1981-1987. 